\title{
Pharmacokinetic study of vinorelbine in Chinese patients with non-small-cell lung cancer by high- performance liquid chromatography (HPLC) with fluorescence detection
}

\author{
Yuchao Dong ${ }^{1 \#}$, Hailiang $\mathrm{Xin}^{2 \#}$, Hui Wang ${ }^{1}$, Qiang $\mathrm{Li}^{1}$, Zhen $\mathrm{Li}^{3}$, Jinhong $\mathrm{Hu}^{3}$, Shen $\mathrm{Gao}^{3}$ and \\ Zhuo Wang ${ }^{3^{*}}$ \\ ${ }^{1}$ Department of Respiratory Medicine, Changhai Hospital, Second Military Medical University, Shanghai 200433, \\ People's Republic of China. \\ ${ }^{2}$ Department of Traditional Chinese Medicine, Changhai Hospital, Second Military Medical University, Shanghai 200433, \\ People's Republic of China. \\ ${ }^{3}$ Department of Pharmacy, Changhai Hospital, Second Military Medical University, Shanghai 200433, \\ People's Republic of China.
}

Accepted 16 July, 2012

\begin{abstract}
To investigate the pharmacokinetics of vinorelbine in Chinese subjects with non-small-cell lung cancer (NSCLC), a high-performance liquid chromatographic method using fluorescence detection was established to determine the concentrations of vinorelbine in human blood and plasma. Samples were collected from 10 Chinese patients with NSCLC after intravenous infusion of $40 \mathrm{mg}$ vinorelbine. Pharmacokinetic parameters were calculated using 3P87 software. The pharmacokinetics of vinorelbine in Chinese patients fitted a two-compartment model. The pharmacokinetic parameters calculated from plasma and blood drug concentrations were: AUC $(530.99 \pm 88.56) \mathrm{ng} \cdot \mathrm{ml}^{-1} \cdot \mathrm{h}$ and $(904.91 \pm 194.97)$ $\mathrm{ng} \cdot \mathrm{ml}^{-1} \cdot \mathrm{h}, \mathrm{C}_{\max }(861.78 \pm 247.25) \mathrm{ng} \cdot \mathrm{ml}^{-1}$ and $(1,053.85 \pm 295.98) \mathrm{ng} \cdot \mathrm{ml}^{-1}$, and $\mathrm{t}_{1 / 2 \beta}(33.70 \pm 1.58) \mathrm{h}$ and $(40.40 \pm 21.30) h$, respectively. The pharmacokinetic profiles of vinorelbine in Chinese NSCLC patients were similar to those reported for non-Chinese NSCLC patients.
\end{abstract}

Key words: Vinorelbine, plasma drug concentration, blood drug concentration, pharmacokinetics, highperformance liquid chromatography.

\section{INTRODUCTION}

Also known as vinorelbine and Navelbine ${ }^{\circledR}$, 5'-noranhydrovinblastine is a first-line, semi-synthetic anticancer agent. It has similar actions to vindesine and vincristine, but lower toxicity than those agents. It has been used widely in China (Liu et al., 2001) and many other countries (Provencio et al., 2011) because of its significant activity in the treatment of advanced non-smallcell lung cancer (NSCLC) and metastatic breast cancer. Many studies have been reported; its pharmacokinetics in cancer patients after single administration (Wargin and 
Lucas, 1994; Levêque and Jehl, 1996; Gebbia and Puozzo, 2005; Robieux et al., 1996) as well as combination with other chemotherapy (Airoldi et al., 2003; Tokudome et al., 2008). Pharmaco- kinetic data were obtained with high-performance liquid chromatographic (HPLC) techniques coupled withfluorescence detection (Gauvin et al., 2000; Robieux et al., 1996), ultraviolet detection (Campone et al., 2001; Puozzo et al., 2007) and electrospray ionization mass spectrometry/mass spectrometry (ESI-MS/MS) (Qian et al., 2011).

Body weight and platelet count were confirmed as influencing blood Clearance of vinorelbine. Also, pharmacokinetic interaction occurred between vinorelbine and other drugs probably due to their inhibition of CYP450-3A4 (Rezai et al., 2011). The results by Robieux et al. (1996) showed lower clearance rate of vinorelbine in patients with severe liver failure but not with moderate secondary liver involvement and supported correspondingly vinorelbine dose reduction. Moreover, association does not alter the pharmacokinetic profile of both vinorelbine and other combined drugs (Airoldi et al., 2003). However, only one Chinese pharmacokinetics study of vinorelbine has been reported (Qian et al., 2011), which using a LC/MS/MS determination method. Therefore, more and further study on the pharmacokinetics of vinorelbine in Chinese would be beneficial for its dose individualization and reduce the side effect.

Here we describe a study of the pharmacokinetics of vinorelbine in Chinese NSCLC patients using a modified HPLC method with fluorescence detection. This HPLC method could fulfill the requirements for a sufficiently simple, accurate and precise assay to carry out pharmacokinetic studies of vinorelbine. The pharmacokinetic results would be the reference for the vinorelbine individual administration and lead to more clinical safety and efficiency.

\section{MATERIALS AND METHODS}

\section{Reagents}

Vinorelbine ditartrate was from Pierre Fabre Corporation (Castres, France). Methanol (BDH, Poole, UK), tetrahydrofuran (Merck, Darmstadt, Germany) and diethyl ether (Merck) were of HPLC grade. Phosphoric acid and potassium dihydrogen phosphate were analytically pure reagents. The water used in all experiments was redistilled.

\section{Equipments}

The chromatographic system consisted of a Shimadzu 10AT pump (Shimadzu, Kyoto, Japan), a Shimadzu RF-10AXL fluorescence detector, a Rheodyne 7725i loading valve fitted with a $100 \mu \mathrm{l}$ sample loop (Rheodyne, Rohnert Park, CA, USA), and a Shimadzu C-R8A integrator. An 80-2 precipitator, a XW-80A vortex mixer, a $\mathrm{pH}$ meter (Orion, Beverly, CA, USA), a JL-120DT ultrasonic cleaner (Shanghai Jili Scientific Instruments, Shanghai, China) and liquid- liquid extraction equipment were used. The excitation wavelength was $280 \mathrm{~nm}$ and the emission wavelength was $360 \mathrm{~nm}$. The HPLC analytical column $(4.0 \mathrm{~mm} \times 150 \mathrm{~mm})$ was packed with $5 \mu \mathrm{m}$ diameter Hypersil- $\mathrm{C}_{18}$ particles (Dalian Elite Analytical Instruments, Dalian, China).

\section{Chromatographic conditions}

The mobile phase was a solution of methanol-phosphate buffertetrahydrofuran $(45: 52.5: 2.5)$. The buffer was prepared using 50 $\mathrm{mM}$ potassium dihydrogen phosphate, and was adjusted to $\mathrm{pH} 4.0$ employing phosphoric acid. Before use, the mobile phase was degassed ultrasonically. The flow rate was $1 \mathrm{ml} \cdot \mathrm{min}^{-1}$. The injection volume was $50 \mu \mathrm{l}$. Chromatography was undertaken at room temperature $\left(\approx 15^{\circ} \mathrm{C}\right)$.

\section{Extraction procedure}

A $5 \mathrm{ml}$ aliquot of diethyl ether was added to $1 \mathrm{ml}$ of plasma sample (or $0.5 \mathrm{ml}$ whole-blood sample diluted with $0.5 \mathrm{ml}$ of water) in a 10 $\mathrm{ml}$ ground-glass stoppered glass centrifuge tube. It was vortexmixed for $3 \mathrm{~min}$, and then centrifuged at $4,000 \mathrm{rpm}$ for $10 \mathrm{~min}$ at room temperature. The supernatant organic phase $(4 \mathrm{ml})$ was transferred to a new glass tube and evaporated to $1 \mathrm{ml}$ at $30^{\circ} \mathrm{C}$. Then, $200 \mu \mathrm{l}$ of potassium dihydrogen phosphate buffer at $\mathrm{pH} 4.0$ was added to it. After vortex-mixing for $2 \mathrm{~min}$ and centrifugation at 4,000 rpm for $10 \mathrm{~min}$ at room temperature, $50 \mu \mathrm{l}$ of the acidic aqueous phase was injected into the chromatographic system.

\section{Precision and extraction recovery}

The chromatogram peak area of vinorelbine was determined for known concentrations of vinorelbine in plasma or blood. Calibration graphs of plasma and blood samples from 1 to $1000 \mathrm{ng} \cdot \mathrm{ml}^{-1}(\mathrm{n}=3)$ were prepared in duplicate by spiking plasma or blood with increasing amounts of vinorelbine to determine the concentration of unknown samples.

Intra-assay precision was determined by analyzing $(n=5)$ plasma or blood samples spiked with vinorelbine at 2,50 and $250 \mathrm{ng} \cdot \mathrm{ml}^{-1}$. Inter-assay precision was tested by analyzing samples of the three concentrations on five days.

The extraction recovery was determined three times at 2, 50 and $250 \mathrm{ng} \cdot \mathrm{ml}^{-1}$. The peak areas obtained after extraction were compared with peaks resulting from standard solutions at the same concentrations.

\section{RESULTS}

\section{Chromatograms}

Chromatograms of vinorelbine in different samples are shown in Figure 1. The retention time of vinorelbine was $8.5 \mathrm{~min}$. There was no obvious interference in plasma or blood samples.

\section{Linearity}

Calibration graphs were obtained by plotting the peak area of vinorelbine against its concentrations in plasma or 


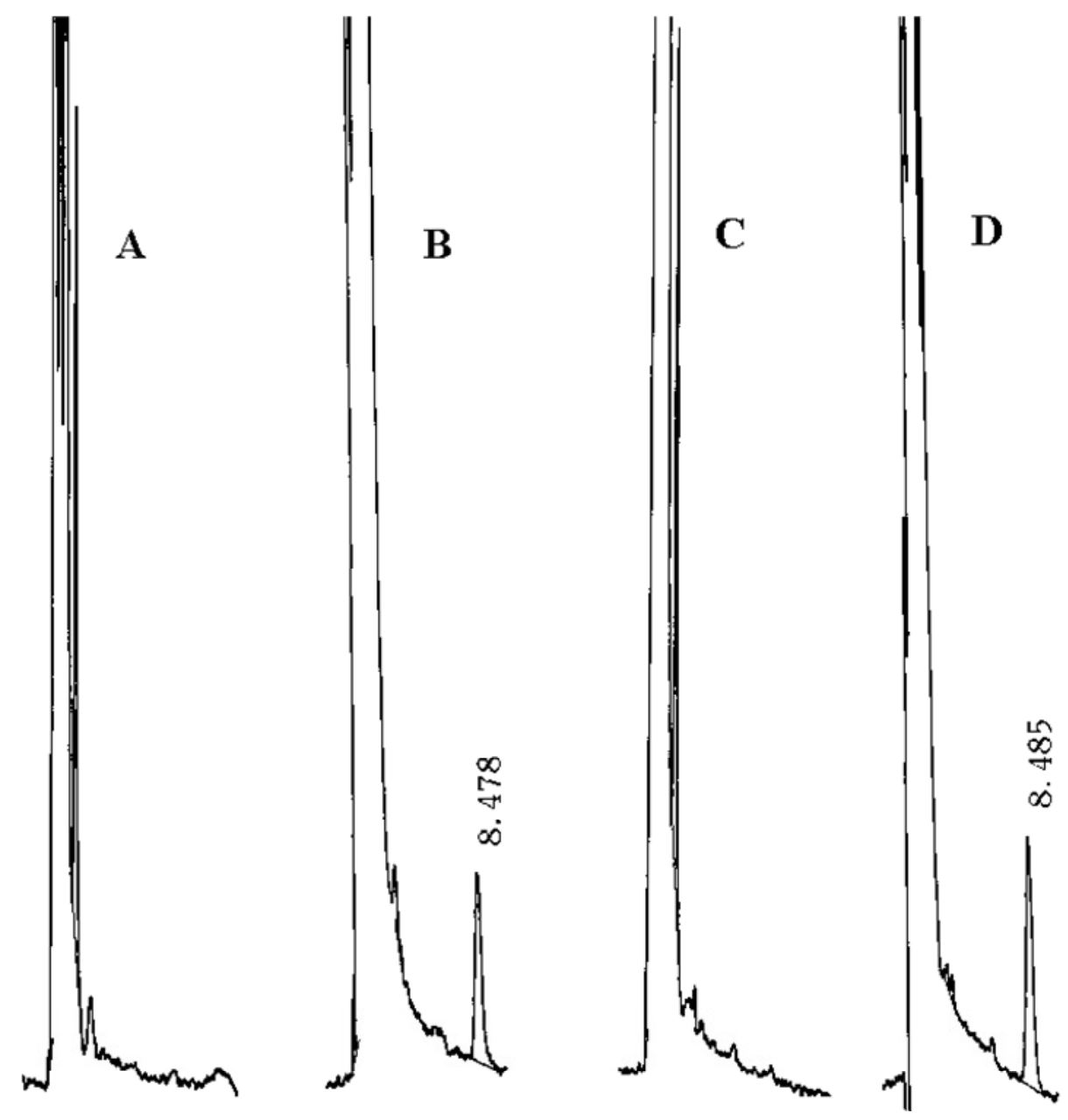

Figure 1. Chromatograms of vinorelbine.

A: blank plasma; B: plasma sample $1 \mathrm{~h}$ after administration of $40 \mathrm{mg}$ vinorelbine; $\mathrm{C}$ : blank blood; D: blood sample $1 \mathrm{~h}$ after administration of $40 \mathrm{mg}$ vinorelbine.

blood. Calibration graphs were set up three times for plasma concentrations of $1,2,5,20,50,100,250$ and $1,000 \mathrm{ng} \cdot \mathrm{ml}^{-1}$ and for blood concentrations of 2, 4, 10, $40,100,500$ and $2,000 \mathrm{ng} \cdot \mathrm{ml}^{-1}$. They were described by the equations of $C=2.76 \times 10^{-4} A+0.8025(r=0.9994)$ for plasma samples and $C=5.34 \times 10^{-4} A+0.4384$ $(r=0.9998)$ for blood samples, where $C$ is the concentration of vinorelbine spiked in blank plasma or blood, and $A$ is the peak area of vinorelbine.

\section{Limit of quantification (LOQ) and limit of detection (LOD)}

The LOQs were $1 \mathrm{ng} \cdot \mathrm{ml}^{-1}$ in plasma and $2 \mathrm{ng} \cdot \mathrm{ml}^{-1}$ in blood, respectively. The LODs were 0.5 and $1 \mathrm{ng} \cdot \mathrm{ml}^{-1}$ (signal-to-noise ratio $(S / N) \geq 3$ ), respectively.

\section{Precision and accuracy}

The results for the accuracy of the determination assay, relative recovery, and extraction recovery are presented in Table 1.

\section{Pharmacokinetic study}

The analytical procedure described above was used to determine the concentrations of vinorelbine in plasma and blood samples from 10 Chinese patients $(7$ males; age $56 \pm 9$ years) with NSCLC. The chemotherapeutic protocol comprised vinorelbine and cisplatin. Vinorelbine (40 mg) was administered as a $10 \mathrm{~min}$ continuous intravenous infusion. Serial blood samples were collected before administration and 1, 6, 18, 24, 48 and $72 \mathrm{~h}$ after infusion. Three milliliters of blood was collected in a 




Figure 2. Concentration-time curve of vinorelbine $(40 \mathrm{mg})$ in 10 Chinese NSCLC patients after rapid intravenous infusion $(n=10)$.

Table 1. Accuracy and recovery of vinorelbine in human plasma and blood $(n=5)$.

\begin{tabular}{ccccc}
\hline $\begin{array}{c}\text { Concentration } \\
\left(\mathbf{n g} \cdot \mathbf{m l}^{-1} \text { ) }\right.\end{array}$ & $\begin{array}{c}\text { RSD Intra-day } \\
(\%)\end{array}$ & $\begin{array}{c}\text { RSD Inter-day } \\
(\%)\end{array}$ & $\begin{array}{c}\text { Relative recovery } \\
(\%) \pm \text { SD }\end{array}$ & $\begin{array}{c}\text { Extraction recovery } \\
(\%) \pm \text { SD }\end{array}$ \\
\hline $\begin{array}{c}\text { Plasma } \\
250\end{array}$ & 2.59 & 4.38 & $103.82 \pm 2.69$ & $77.52 \pm 3.39$ \\
50 & 6.53 & 6.72 & $98.77 \pm 6.45$ & $70.92 \pm 4.63$ \\
2 & 7.91 & 10.79 & $101.69 \pm 8.04$ & $74.41 \pm 8.03$ \\
& & & & \\
Blood & & & & \\
250 & 3.21 & 4.57 & $100.03 \pm 3.21$ & $75.37 \pm 2.42$ \\
50 & 6.24 & 7.21 & $102.82 \pm 6.42$ & $71.28 \pm 4.45$ \\
2 & 8.54 & 9.91 & $97.11 \pm 8.29$ & $91.77 \pm 7.84$ \\
\hline
\end{tabular}

RSD: relative standard deviation

heparinized glass tube. A total of $0.5 \mathrm{ml}$ was taken for a blood sample; the rest was prepared for a $1 \mathrm{ml}$ plasma sample after centrifugation at $4,000 \mathrm{rpm}$ for $5 \mathrm{~min}$ at room temperature.

Figure 2 shows the mean plasma and blood concentration versus time profiles of vinorelbine in 10 NSCLC patients. Pharmacokinetic parameters (Table 2) were estimated using 3P87 pharmacokinetic software (Chinese Pharmacological Society, Beijing, China). The pharmacokinetic characteristics of vinorelbine in Chinese NSCLC patients afforded a two-compartment model calculated by using plasma or blood concentration data. The elimination half-life of vinorelbine was $33.70 \pm 1.58 \mathrm{~h}$ for plasma and $40.40 \pm 21.30 \mathrm{~h}$ for blood samples. We used values of the area under the curve (AUC) to calculate the ratio $A \cup C_{\text {blood }} / A \cup C_{\text {plasma, }}$ which was 1.7. It was comparable with the ratio of blood concentration /plasma concentration in these 10 patients, remaining almost constant $(\approx 1.7)$ during the $72 \mathrm{~h}$ of the study.

\section{DISCUSSIONS}

The pharmacokinetic information of vinorelbine as firstline chemotherapy in Chinese NSCLC patients is too few (Qian et al., 2011). In this paper, we modified a HPLC 
Table 2. Pharmacokinetic parameters obtained from plasma and blood concentrations of 10 Chinese NSCLC patients after rapid intravenous infusion of $40 \mathrm{mg}$ vinorelbine (mean \pm S.D., $n=10)$.

\begin{tabular}{cccc}
\hline Parameter & Unit & Plasma & Blood \\
\hline$A$ & $\mathrm{ng} \cdot \mathrm{ml}^{-1}$ & $855.53 \pm 248.04$ & $1041.95 \pm 299.92$ \\
$\alpha$ & $\mathrm{h}^{-1}$ & $3.82 \pm 0.2$ & $3.25 \pm 0.15$ \\
$B$ & $\mathrm{ng} \cdot \mathrm{ml}^{-1}$ & $6.24 \pm 1.6$ & $11.89 \pm 7.64$ \\
$\beta$ & $\mathrm{h}^{-1}$ & $0.02 \pm 0.00$ & $0.02 \pm 0.01$ \\
$V_{d}$ & $\mathrm{~L}$ & $49.15 \pm 12.86$ & $39.86 \pm 9.09$ \\
$t_{1 / 2 \alpha}$ & $\mathrm{h}$ & $0.18 \pm 0.01$ & $0.21 \pm 0.01$ \\
$t_{1 / 2 \beta}$ & $\mathrm{h}$ & $33.7 \pm 1.58$ & $40.4 \pm 21.3$ \\
$K_{12}$ & $\mathrm{~h}^{-1}$ & $2.16 \pm 0.53$ & $2.00 \pm 0.45$ \\
$K_{21}$ & $\mathrm{~h}^{-1}$ & $0.05 \pm 0.01$ & $0.06 \pm 0.04$ \\
$K_{10}$ & $\mathrm{~h}^{-1}$ & $1.63 \pm 0.38$ & $1.21 \pm 0.41$ \\
$A U C$ & $\mathrm{ng} \cdot \mathrm{ml}^{-1} \cdot \mathrm{h}$ & $530.99 \pm 88.56$ & $904.91 \pm 194.97$ \\
$C L s$ & $\mathrm{~L} \cdot \mathrm{h}^{-1}$ & $77.25 \pm 15.37$ & $46.14 \pm 12.07$ \\
\hline$A$
\end{tabular}

$A$, coefficient of exponential functions of distribution phase; $\alpha$, absorption rate constant; $B$, coefficient of exponential functions of elimination phase; $\beta$, elimination rate constant; $V_{d}$, volume of distribution; $t_{1 / 2 \alpha}$, half-life of absorption; $t_{1 / 2 \beta}$, half-life of elimination; $K_{12}$, rate constant from central-compartment to peripheral-compartment; $K_{21}$, rate constant from peripheralcompartment to central-compartment; $K_{10}$, elimination rate constant from centralcompartment; AUC, area under the concentration-time curve; CLs, clearance.

method (Gauvin et al., 2000; Robieux et al., 1996) by using a less expensive mobile phase (comprising mainly methanol and phosphate buffer) and sensitive fluorescence detection to determine the vinorelbine concentration in both plasma and blood. Fluorescence detection is certainly more routinely usable detection mode for drugs monitoring. An in-vitro study (Urien et al., 1993) reported that $84 \%$ of vinorelbine is bound to blood cells, mainly to platelets (78\%). Hence, we also determined the blood concentration in patients to compare with the literature (Gauvin et al., 2000). It was reported that vinorelbine concentration in blood may remain constant, but that in plasma would vary depending on differences in the centrifuge conditions (Puozzo et al., 2007). So determination of the concentrations in both blood and plasma meanwhile is more significant.

The present method was relatively easy and feasible to perform and allowed to determine vinorelbine in blood and plasma at the nanogram level. Also, we employed two steps of careful vortex-mixing of overall duration 5 min instead of two steps of speed agitation for $60 \mathrm{~min}$ as commonly adopted procedure (Gauvin et al., 2000). Thus the whole procedure of sample extraction could save $55 \mathrm{~min}$, which made the determination more rapid. Although we did not use an internal standard to simplify the pretreatment procedure, the results showed relatively sensitivity and accuracy and were successfully applied to pharmacokinetic studies.

The pharmacokinetic profile of vinorelbine is often described as a 3-compartment model characterised by a long terminal half-life $\left(t_{1 / 2}\right)$ that varies between 20 and 40 h, a large apparent volume of distribution $(\mathrm{Vd})$ of around $70 \mathrm{~L} / \mathrm{kg}$ and a high plasma clearance (CL) between 72.54 and $89.46 \mathrm{~L} / \mathrm{h}$ when determined by HPLC method (Wargin and Lucas, 1994; Levêque and Jehl, 1996).

The disposition of the alkaloid is not altered by concurrent co-administration of cisplatin (Wargin and Lucas, 1994; Levêque and Jehl, 1996; Delord et al., 2009). However, Gauvin et al. (2000) reported the pharmacokinetic profiles exhibiting a three-compartment model with a mean elimination half-life of $42 \mathrm{~h}$. The calculated pharmacokinetic parameters (Table 2) in our study were consistent with these previous study (Wargin and Lucas, 1994; Levêque and Jehl, 1996), with the mean elimination half-life was $33.70 \pm 1.58 \mathrm{~h}$ for plasma and $40.40 \pm 21.30 \mathrm{~h}$ for blood, in spite of using a simple HPLC method.

\section{Conclusion}

The pharmacokinetic characteristics of vinorelbine in Chinese NSCLC patients were similar to those of nonChinese patients and non-NSCLC patients described in the literature. Based on our and previous findings, dose modifications of vinorelbine of first use in Chinese patients may need not be under consideration generally. Our study may provide the reference for the vinorelbine individual administration and bring more clinical safety and efficiency to Chinese patients. However, more and further study may need to confirm our results. 


\section{ACKNOWLEDGMENTS}

This research was supported by the National Natural Science Foundation of China (grant number 30973593). The help of Professor Guo-Rong Fan during the experiments is especially appreciated.

\section{REFERENCES}

Airoldi M, Cattel L, Cortesina G, Giordano C, Passera R, Pedani F, Novello S, Bumma C, Gabriele P (2003). Gemcitabine and vinorelbine in recurrent head and neck cancer: pharmacokinetic and clinical results. Anticancer Res. 23:2845-2852.

Campone M, Fumoleau P, Delecroix V, Deporte-Fety R, Perrocheau G, Vernillet L, Borg-Olivier O, Louboutin JP, Bissery MC, Riva A, Azli N (2001). Phase I dose-finding and pharmacokinetic study of docetaxel and vinorelbine as first-line chemotherapy for metastatic breast cancer. Ann. Oncol. 12:909-918.

Delord JP, Puozzo C, Lefresne F, Bugat R (2009). Combination chemotherapy of vinorelbine and cisplatin: a phase I pharmacokinetic study in patients with metastatic solid tumors. Anticancer Res. 29:553-560

Gauvin A, Pinguet F, Poujol S, Astre C, Bressolle F (2000). Highperformance liquid chromatographic determination of vinorelbine in human plasma and blood: application to a pharmacokinetic study. J. Chromatogr. B. 748:389-399.

Gebbia V, Puozzo C (2005). Oral versus intravenous vinorelbine: clinical safety profile. Expert. Opin. Drug Saf. 4:915-928.

Levêque D, Jehl F (1996). Clinical pharmacokinetics of vinorelbine. Clin. Pharmacokinet. 31:184-197.

Liu CY, Sun YH (2001). Present situation of second-line chemotherapy for non-small cell lung cancer. Shi Yong Ai Zheng Za Zhi. pract. J. cancer (in Chinese) 16:329-336.

Provencio M, Isla D, Sánchez A, Cantos B (2011). Inoperable stage III non-small cell lung cancer: Current treatment and role of vinorelbine. J. Thorac. Dis. 3:197-204.

Puozzo C, Ung HL, Zorza G (2007). A high performance liquid chromatography method for vinorelbine and 4-O-deacetyl vinorelbine: a decade of routine analysis in human blood. J. Pharm. Biomed. Anal. 44:144-149.
Qian J, Wang Y, Chang J, Zhang J, Wang J, Hu X (2011). Rapid and sensitive determination of vinorelbine in human plasma by liquid. $J$. Chromatogr. B. 879:662-668.

Rezai K, Urien S, Isambert N, Roche H, Dieras V, Berille J, Bonneterre $\mathrm{J}$, Brain $\mathrm{E}$, Lokiec $\mathrm{F}$ (2011). Pharmacokinetic evaluation of the vinorelbine-lapatinib combination in the treatment of breast cancer patients. Cancer Chemother. Pharmacol. 68:1529-1536.

Robieux I, Sorio R, Borsatti E, Cannizzaro R, Vitali V, Aita P, Freschi A, Galligioni E, Monfardini S (1996). Pharmacokinetics of vinorelbine in patients with liver metastases. Clin. Pharmacol. Ther. 59:32-40.

Robieux I, Vitali V, Aita P, Freschi A, Lazzarini R, Sorio R (1996). Sensitive high-performance liquid chromatographic method with fluorescence detection for measurement of vinorelbine plasma concentrations. J. Chromatogr. B. 675:183-187.

Tokudome N, Ito Y, Hatake K, Toi M, Sano M, Iwata H, Sato Y, Saeki T, Aogi K, Takashima S (2008). Trastuzumab and vinorelbine as firstline therapy for HER2-overexpressing metastatic breast cancer: multicenter phase II and pharmacokinetic study in Japan. Anticancer Drugs 19:753-759.

Urien S, Bree F, Breillout F, Bastian G, Krikorian A, Tillement JP (1993). Vinorelbline high-affinity binding to human platelets and lymphocytes: distribution in human blood. Cancer Chemother. Pharmacol. 32:231234.

Wargin WA, Lucas VS (1994). The clinical pharmacokinetics of vinorelbine (Navelbine). Semin. Oncol. 21:21-27. 